\title{
Generalized Aggressive Periodontitis and Correlated Factors: An Histomorphometric Study
}

\section{Barbara Buffoli ${ }^{* \circ}$, Gianluca Garzetti $^{{ }^{\circ}}$, Stefano Calza $^{3}$, Eleonora Scotti ${ }^{2}$, Elisa Borsani ${ }^{1}$, Veronica Cappa ${ }^{3-4}$, Lia Remondini' ${ }^{5}$, Magda Mensi²}

${ }^{1}$ Section of Anatomy and Physiopathology, Department of Clinical and Experimental Sciences, University of Brescia, Brescia, Italy.

${ }^{2}$ Section of Periodontics, School of Dentistry, Department of Surgical Specialties, Radiological Science and Public Health, University of Brescia, Brescia, Italy.

${ }^{3}$ Department of Molecular and Translational Medicine, University of Brescia, Brescia, Italy.

${ }^{4}$ Big\&Open Data Innovation Laboratory (BODaI-Lab), University of Brescia, Brescia, Italy.

${ }^{5}$ Department of Health Sciences, University of Piemonte Orientale “UPO”, Italy

${ }^{\circ}$ These authors contributed equally

\section{*Corresponding Author:}

Section of Anatomy and Physiopathology, Department of Clinical and Experimental Sciences, University of Brescia, Brescia, Italy. Phone +390303717479; Fax +390303717486; e-mail: barbara.buffoli@unibs.it

Running title: Generalized aggressive periodontitis: an histomorphometric study

Key words: Generalized aggressive periodontitis, histomorphometric analysis, smoke, plaque, aging, Pocket Probing Depth (PPD). 


\section{Abstract}

Background: Periodontitis is a disease that leads to serious functional and aesthetic dysfunctions resulting in impairment of life quality; it exists in different forms and its etiology is related with multiple component causes. Angiogenesis, together with inflammatory infiltrate, are two key processes in the evolution of inflammatory processes involving gingival tissues. The aim of this study was to evaluate their alterations in relation to some important periodontal diseases-associated factors (i.e. smoke, sex, age, plaque, pus, probing pocket depth) in patients with diagnosis of Generalized Aggressive Periodontitis (GAP).

Methods: Eighteen subjects with GAP were enrolled for this study. Percentage of inflammatory cells and vascular area were measured and evaluated in relation with each periodontal diseases-associated factor.

Results: The results showed a significant decrease of the percentage of vascular area in association with almost all the periodontal diseases-related factors (smoke, age, PPD, plaque) and not significant differences in the percentage of inflammatory cells.

Conclusions: Data suggest that, even if these factors are often commonly related to worsening of periodontal status, they did not affect inflammatory and vascular pattern, as we can assume. For the future, additional studies, aimed to evaluated each singular factor in a wider sample size, should be planned. 


\section{Introduction}

Periodontitis is a disease that, if untreated, leads to serious functional and aesthetic impairments, as well as a strong conditioning of social life, resulting in impairment of life quality.

The phenotype is characterized by an exaggerated, not very effective and not resolving, inflammation of the periodontal connective tissue, leading to the periodontal destruction, due to a bacterial infection [1]. Severe periodontitis, if not treated, can result in impaired occlusion, occasional pain and discomfort and, eventually, tooth loss [2].

In 1999, the "International Workshop for a Classification of Periodontal Diseases and Conditions" classified disorders that affect the supporting tissues of teeth into eight main categories [3]. Aggressive Periodontitis (AP) may occur in two different forms: localized and generalized. Localized forms affect the first molar/incisor teeth with interproximal attachment loss on at least two permanent teeth (one of which is a first molar) and involving no more than two teeth other than first molars and incisors. Differently, in Generalized Aggressive Periodontitis (GAP), tissue loss may begin to occur at the permanent first molars and incisors, because these are the first teeth to erupt. Then, the disease progresses rapidly, so that most or all the permanent teeth were involved in both severe attachment and bone loss [4].

GAP is a form of periodontitis with a relatively low prevalence in population [5]. Diagnosis is associated with several diagnostic criteria: early age of onset, loss of periodontal tissue at multiple permanent teeth, clinically and radiographically detectable periodontal destruction, relatively high progression rate of periodontal tissue loss, not common early teeth exfoliation, systemically healthy patients.

About the etiology of this pathology, periodontitis is a complex disease with multiple component causes, some with their basis in genetics, some caused by epigenetic influences and others that are modifiable because they are related to patient behaviors, medications or environmental factors, all of which conspire to establish and propagate the periodontal lesion. In addition to such 'patient-specific' 
risk factors, there are also 'site-specific characteristics' (e.g. anatomical factors), which may favor the development of a lesion [1].

Periodontal lesions induce tissue changes both inside the gum and the alveolar bone. Continuous action of etiological factors, which induce and sustain pathological changes in periodontal disease, may induce irreversible changes. Angiogenesis, together with inflammatory infiltrate, may be important in the evolution of inflammatory processes involving gingival tissues [6-7]. During gingival inflammation, in fact, the cell infiltrate occupies an increasing proportion of the connective tissue and might cause a collagen degradation or a fibrotic reaction [8-9] by stimulating effects of inflammatory mediators of the connective tissue [10-11]. In addition, inflammation is associated with the activation of pathological angiogenesis and a high number of newly-formed blood vessels, quantified as microvessel density (MVD) [7,12].

Considering the involvement of these two processes in the pathogenesis of GAP, the aim of our study was to evaluate their alterations in relation to some important factors (smoke, sex, age, plaque, pus, PPD (Probing Pocket Depth), known as periodontal diseases-associated factors. In particular our ipothesys has been to find a statistically significant major mean percentage of vascular area and inflammatory cells in smokers patients. AgP patients have been chosen to better define histologic characteristics of a low prevalence illness. 


\section{Materials and Methods}

\section{Study design and ethical approval}

This observational study was conducted at the Dental Clinic "Lidia Verza", University of Brescia, Italy, from January 2014 to November 2016. The study protocol was reviewed and approved by the the Ethical Committee of “AO Spedali Civili” Hospital of Brescia (protocol n ${ }^{\circ} 1473$ ) and conducted in accordance with the Helsinki Declaration. Written informed consent was obtained from all patients.

\section{Patients and collection of the samples}

Eighteen subjects were enrolled for this study. The inclusion criteria were: age older than 18 years; no systemic illnesses or disorders; no medical treatment that may impair healing (immunodepression, immunosuppression, diabetes, etc.); diagnosis of GAP [5]; presence of at least 5 teeth in each quadrant; ability and willingness to give written informed consent.

The exclusion criteria were: pregnant or breastfeeding women; women practicing birth control methods; allergy or other severe adverse reactions to amoxicillin and metronidazole; use of local and/or systemic antibiotics in the 6 months preceding the beginning of the study.

During clinical assessments, age, gender (male/female), smoking habits (smoker/non-smoker), clinical and dental history were collected before the beginning of the treatment. One calibrated blinded examiner evaluated Pocket Probing Depth (PPD), Clinical Attachment Level (CAL), Gingival Margin Recession (GMR), Bleeding on Probing (BoP), Plaque Index (PI) using a periodontal probe with a diameter of 0.5 millimeters (UNC 15, Hu-Friedy Italy, Milan, Italy).

The collection of the samples was carried out in all cases after topical anesthesia with $2 \%$ mepivacaine and epinephrine 1:100.000.

Hopeless or useless to treat teeth were selected. Clinical data were collected and 6 sites for tooth were tested. Gingival biopsies $(2 \mathrm{~mm} \times 2 \mathrm{~mm})$ from the attached tissue around a selected natural tooth were taken by scalpel (15C, Hu-Friedy Italy, Milan, Italy) from the gingival margin of deepest sites ( $\mathrm{PPD} \geq 5 \mathrm{~mm}$ ). Tissue sample were fixed in $10 \%$ buffered formalin $(\mathrm{pH} 7.0-7.2)$ for 24 hours, conventionally dehydrated and embedded in paraffin wax according to standard procedure. Serial 
sections ( $7 \mu \mathrm{m}$ thick) were cut using a microtome (Microm HM 325, Thermo Scientific, Walldorf, Germany) and collected on poly-l-lysine coated glass slides.

\section{Histomorphometric analysis}

Sections were deparaffinized in xylene, rehydrated in descending concentrations of ethanol, water and stained with Hematoxylin-Eosin staining (standard protocol) and Masson-Goldner Trichrome staining (Merck KGaA, Darmstadt, Germany) for the evaluation of the percentage of inflammatory cells and the percentage of vascular area (Figure 1).

In order to quantify the percentage of inflammatory cells and the percentage of vascular area, digitally fixed images (standardized arbitrary area) for each section ( 5 serial sections/sample) was evaluated using an optical light microscope (Olympus BX50, Olympus, Hamburg, Germany) at a final

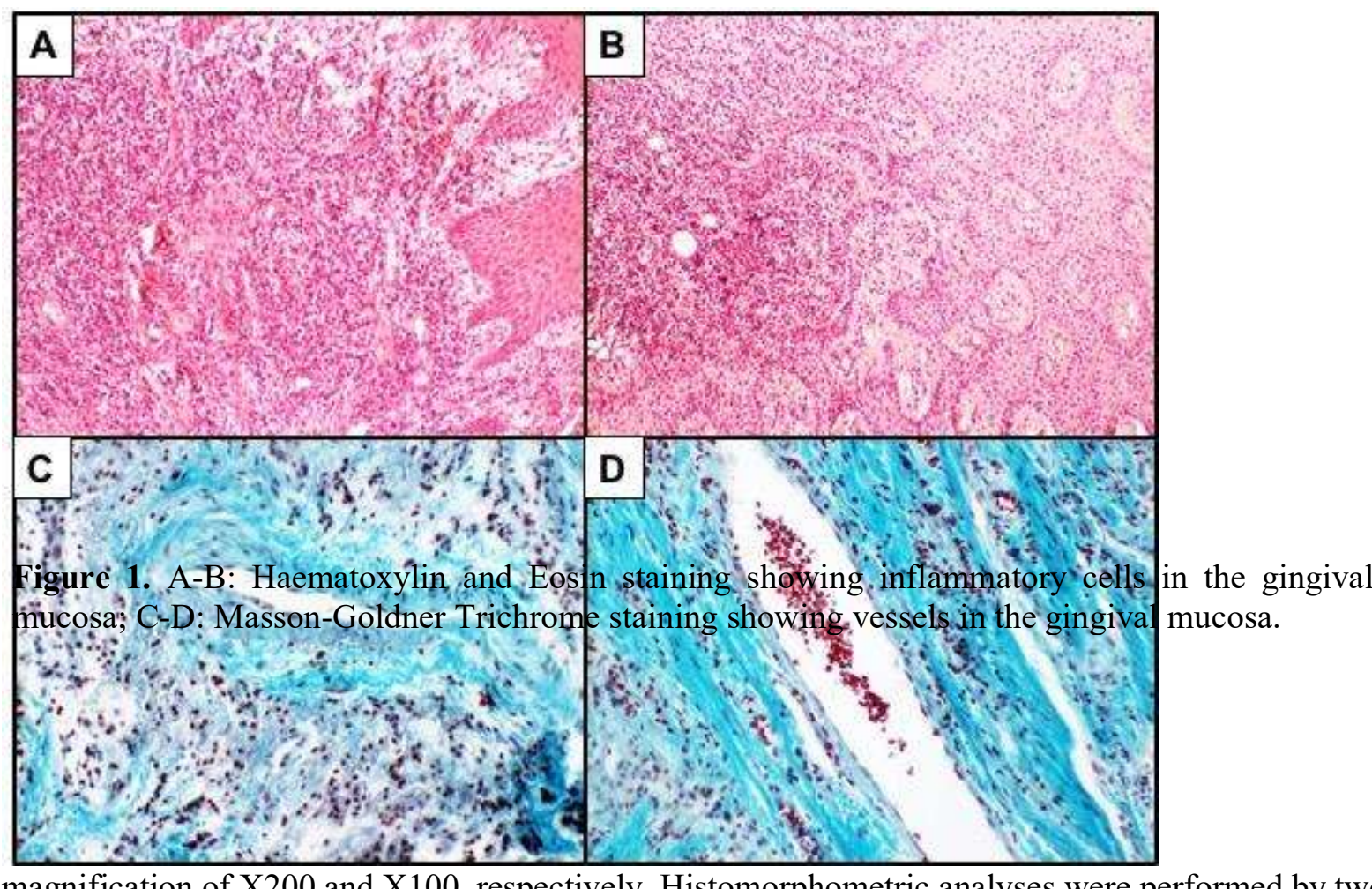

magnification of X200 and X100, respectively. Histomorphometric analyses were performed by two blinded investigators. Quantitative analysis of the percentage of inflammatory cells and vascular area was performed using a digital color video camera equipped with an image analysis program (Image Pro Premier 9.1; Immagini e Computer, Milan, Italy). 


\section{Statistical analysis}

As percentage of non-smokers who generally attends the clinic is about $60 \%$, sample size was calculated assuming a 2 vs 3 ratio between arms (smokers vs non-smokers). A sample size of 18 subjects (11 non-smokers and 7 smokers) is sufficient to detect a clinically important difference of $0.45 \%$ between groups in reducing vascular area, assuming a mean in healthy and non-smoking patients of $1 \%$ [15], a common standard deviation of $0.3 \%$ with $80 \%$ power and a $5 \%$ level of significance..

Similarly, as mean of inflammatory infiltrate reported by literature in healthy and non-smoking patients is $9 \%$ with a standard deviation of $1.5 \%$ [15], assuming a $2.5 \%$ reduction in smokers (mean $6 \%$ ) a sample size of 16 patients (10 non-smokers and 6 smokers) will deliver a $80 \%$ power at a $5 \%$ level of significance.

In order to take into account both aims, 18 patients were enrolled for this study.

Binary coded variable BoP was summarised as counts and percentages. Continuous variables such as PPD and CAL loss were summarised as geometric mean and standard deviation (sd). Due to the high number of zeros, gingival recession was summarised both as the percentage of zero values as well as the geometric mean and sd of non zero values.

Laboratory data on inflammatory infiltrate and alveolar areas were recorded as within patient averages and standard deviations; therefore statistical analyses accounted for measurement precision using inverse variance weighting. We used univariate linear regression models to evaluate the relationship between log transformed outcomes variables (inflammatory infiltrate and vascular area) and clinical determinants: gender (male/female), age (coded as $<51$ and $\geq 51$ years-old), smoking habits (smoker/non-smoker), PPD (coded as $<9 \mathrm{~mm}$ and $\geq 9 \mathrm{~mm}$ ), presence of plaque on tooth surface (yes/no) and pus (yes/no). Results were reported as estimates and 95\% confidence intervals. All 
analysis were performed assuming a significance level of 5\% using the statistical software $\mathrm{R}$ (version 3.4.2). Sample size was computed using $\mathrm{G}^{*}$ Power (version 3.1).

\section{Results}

Out of 18 patients, $72 \%$ were females, $55.6 \%$ were more than 51 years old and smokers. About twothirds of subjects had plaque on tooth surface and pus. Full mouth means of PPD, CAL and GMR (different from 0) and BOP were respectively $3.46 \mathrm{~mm}, 4.03 \mathrm{~mm}$ and $2.39 \mathrm{~mm}$, and 49\% (Table 1).

Considering vascular area, young patients showed a significant increase compare to older ones $(0.39 \%$ vs $0.28 \%$, p-value $=0.008)$ and was two times higher in non-smokers compared to smokers $(0.45 \%$ vs $0.24 \%, \mathrm{p}$-value $=0.038)$. Moreover, subjects with a PPD at baseline higher than $9 \mathrm{~mm}$

Table 1. Clinical characteristics of patients (total mouth).

\begin{tabular}{lc} 
& Mean (sd) \\
\hline PPD $(\mathrm{mm})$ & $3.46(1.79)$ \\
CAL Loss $(\mathrm{mm})$ & $4.03(1.84)$ \\
Gingival recession $(\neq$ & $2.39(1.73)$ \\
$0)$ & $\%$ \\
\hline Gingival recession $=0$ & 74.6 \\
Bleeding on Probing & 49.0 \\
\hline
\end{tabular}

showed a reduced vascular area compared with baseline PPD lower than $9 \mathrm{~mm}(0.22 \%$ vs $0.40 \%$, pvalue $=0.004)$. Vascular area was also more than halved in subject with residual plaque on tooth surfaces $(0.23 \%$ vs $0.57 \%$, p-value $=0.008)$. No determinant showed a statistically significant association with inflammatory infiltrate through females showed a slight and marginally significant $(p=0.07)$ increase in infiltration area $(4.81 \%)$ compared to males $(2.62 \%)$, see Table 2. 


\section{Discussion}

In this study, we evaluated the influence of some periodontal disease-associated factors (i.e. smoking, age, PPD, plaque, pus, sex) respect to vascular density and inflammatory response. The data obtained showed a significant decrease in the percentage of vascular area in relation with age, smoking, PPD and plaque. On the contrary, the percentage of inflammatory cells showed a similar but not significant trend.

Table 2. Mean percentage (and 95\%CI) of inflammatory cells and vascular area. *Raw log-means were estimated weighting for inverse of variance within patient and then transform through exponential function.

\begin{tabular}{|c|c|c|}
\hline & $\begin{array}{l}\text { Mean percentage of } \\
\text { inflammatory cells }\end{array}$ & $\begin{array}{c}\text { Mean percentage of } \\
\text { vascular area }\end{array}$ \\
\hline Overall raw weighted mean* & $4.38 \%$ & $0.24 \%$ \\
\hline $\begin{array}{r}\text { Female } \\
\text { Male } \\
p \text {-value }\end{array}$ & $\begin{array}{c}4.81 \%[3.70 ; 6.25] \\
2.62 \%[1.42 ; 4.84] \\
0.071\end{array}$ & $\begin{array}{l}0.24 \%[0.20 ; 0.28] \\
0.46 \%[0.22 ; 0.60] \\
0.088\end{array}$ \\
\hline $\begin{array}{r}<51 \text { yrs old } \\
\geq 51 \text { yrs old } \\
p \text {-value }\end{array}$ & $\begin{array}{c}4.38 \%[3.16 ; 6.07] \\
4.39 \%[2.76 ; 6.99] \\
0.990\end{array}$ & $\begin{array}{c}0.39 \%[0.27 ; 0.54] \\
0.28 \%[0.20 ; 0.26] \\
0.008\end{array}$ \\
\hline $\begin{array}{l}\text { Smoking habits } \\
\qquad \begin{array}{r}\text { Non-smokers } \\
\text { Smokers } \\
p \text {-value }\end{array}\end{array}$ & $\begin{array}{c}4.82 \%[3.44 ; 6.76] \\
3.82 \%[2.45 ; 5.75] \\
0.367\end{array}$ & $\begin{array}{c}0.45 \%[0.25 ; 0.80] \\
0.24 \%[0.20 ; 0.27] \\
0.038\end{array}$ \\
\hline $\begin{array}{r}<9 \\
\geq 9 \\
p \text {-value }\end{array}$ & $\begin{array}{c}4.33 \%[3.03 ; 6.20] \\
4.45 \%[2.98 ; 6.65] \\
0.920\end{array}$ & $\begin{array}{c}0.40 \%[0.29 ; 0.55] \\
0.22 \%[0.20 ; 0.26] \\
0.004\end{array}$ \\
\hline $\begin{array}{r}\text { Yes } \\
\text { No } \\
p \text {-value }\end{array}$ & $\begin{array}{c}6.13 \%[2.38 ; 15.8] \\
4.26 \%[3.2 ; 5.6] \\
0.466\end{array}$ & $\begin{array}{l}0.23 \%[0.20 ; 0.27] \\
0.57 \%[0.31 ; 1.04] \\
0.008\end{array}$ \\
\hline Pus & & \\
\hline
\end{tabular}


With this assumption, we decided to discuss only the percentage of vascular area and for simplifying the comprehension of the results, we discussed it factor by factor.

\section{Smoking}

Tobacco and its oxidation products are involved in the modulation of periodontal disease progression and in the alteration of its clinical features. Prakash et al. [13] reported alterations in the histological pattern related to vascular density, showing a significantly decrease of microvascular density in smokers with periodontitis respect to non-smokers. Our results supported these data confirming a significantly decrease in the percentage of vascular area in smoker patients affected by GAP [13-14]. Aging

Our results showed a significantly decrease in the percentage of vascular area in patients aged over 50 years. These data are in accordance with Preshaw et al. [15], who asserted that the effectiveness of the immune system in old age is compromised by physiological changes, where there is decreased blood vessel density with increasing age.

\section{Pocket Probing Depth (PPD)}

Periodontal pocket is defined as a pathologically deepened gingiva sulcus, according to the definition reported by the American Academy of Periodontology. It occurs with destruction of supporting periodontal tissue and migration of alveolar bone, periosteum and periodontal ligament versus the tooth apex.

Vascular density of marginal gingiva is supported by arteries that extend into the periodontal ligament and into the alveolar bone and periosteum [16]. Consequently, vascularisation of marginal gingiva should be more altered in relation with the pocket depth. This concept is supported by our results, which showed a significant decrease of the mean percentage of the vascular area when PPD $\geq 9 \mathrm{~mm}$ was recorded. 


\section{Plaque}

The percentage of vascular area were higher in no plaque then plaque group. These data were difficult to explain. However, since we observed a concomitant decrease of the percentage of inflammatory cells, a possible explanation was suggested by Sreedevi et al. [17], who reported that the suppression of vascular inflammatory reaction could indicate an impairment of the defense mechanisms within the tissues and a more susceptibility to plaque infection. It means that an higher vascular area in this kind of susceptible patients could not be related to a relevant quantity of plaque at level site.

Pus

Considering the presence/absence of pus, no significant differences were observed. However, this factor should be more investigated.

$\operatorname{Sex}$

Women showed a higher percentage of inflammatory cells respect to men and a not significant reduction in the percentage of vascular area. In literature sex differences in periodontal diseases was reported. The Authors affirmed that men appeared at greater risk for periodontal disease than women; on the contrary, men do not appear at higher risk for more rapid periodontal destruction than women [18]. This difference could be considered to understand our results; however, additional studies aim to evaluate the incidence of sex in the characterization of the vascular and inflammatory patterns of periodontal disease should be conducted. 


\section{Conclusion}

It is known that during gingival inflammation the cell infiltrate occupies an increasing proportion of the connective tissue [8-9] and inflammation is associated with the activation of pathological angiogenesis and a high number of newly-formed blood vessels [7,12]. In this study, we did not compare the percentage of vascular area and inflammatory cells with healthy patients but we evaluated how some factors influenced these percentages in GAP.

Taking together our results showed a significant decrease of the percentage of vascular area in association with almost all the periodontal diseases-related factors (smoke, age, PPD, plaque) and not significant differences in the percentage of inflammatory cells. It could suggest that, even if these factors are often commonly related to worsening of periodontal status, they did not affect inflammatory and vascular pattern, as we can assume. So, for the future, additional studies, aimed to evaluated each singular factor in a wider sample size, should be planned. 


\section{References}

[1] J. Meyle, I. Chapple, "Molecular aspects of the pathogenesis of periodontitis," Periodontol 2000, vol. 69 , no. 1 , pp. $7-17,2015$.

[2] B.L. Pihlstrom, B.S. Michalowicz, N.W. Johnson, "Periodontal diseases," Lancet, vol. 366, no. 9499, pp. 1809-1820, 2005.

[3] J.M. Albandar, L.J. Brown, J.A. Brunelle, H. Löe, "Gingival state and dental calculus in earlyonset periodontitis," J Periodontol, vol. 67, no. 10, pp. 953-959, 1996.

[4] J.M. Albandar, "Aggressive periodontitis: case definition and diagnostic criteria," Periodontol 2000, vol. 65, no.1, pp. 13-26, 2014.

[5] C. Susin, A.N. Haas, J.M. Albandar, "Epidemiology and demographics of aggressive periodontitis," Periodontol 2000, vol. 65, no. 1, pp. 27-45, 2014.

[6] R. Cornelini R, L. Artese, C. Rubini, M. Fioroni, G. Ferrero, A. Santinelli, A. Piattelli, "Vascular endothelial growth factor and microvessel density around healthy and failing dental implants," Int $J$ Oral Maxillofac Implants, vol. 16, no. 3, pp. 389-393, 2001.

[7] A. Kasprzak, A. Surdacka, M. Tomczak, M. Konkol, "Role of high endothelial postcapillary venules and selected adhesion molecules in periodontal diseases: a review," J Periodontal Res, vol. 48, no. 1, pp. 1-21, 2013.

[8] E. Borsani, S. Salgarello, M. Mensi, R. Boninsegna, A. Stacchiotti, R. Rezzani R, P. Sapelli, R. Bianchi, L.F. Rodella, "Histochemical and immunohistochemical evaluation of gingival collagen and metalloproteinases in peri-implantitis," Acta Histochem, vol. 107, no. 3, pp. 231-240, 2005.

[9] B. Buffoli, M. Dalessandri, G. Favero, M. Mensi, D. Dalessandri, F. Di Rosario, C. Stacchi, R. Rezzani, S. Salgarello, L.F. Rodella, “AQP1 expression in human gingiva and its correlation with periodontal and peri-implant tissue alterations," Acta Histochem, vol. 116, no. 4, pp. 898-904, 2014. [10] R.C. Page, H.E. Schroeder, "Pathogenesis of inflammatory periodontal disease. A summary of current work," Lab Invest, vol. 34, no. 3, pp. 235-249, 1976. 
[11] R. Younes, C. Ghorra, S. Khalife, S. Igondjo-Tchen-Changotade, M. Yousfi, C. Willig, K. Senni, G. Godeau, N. Naaman, "Pertinent cell population to characterize periodontal disease," Tissue Cell, vol. 41, no. 2, pp. 141-150, 2009.

[12] M. Vladau, A.M. Cimpean, R.A. Balica, A.A. Jitariu, R.A. Popovici, M. Raica, "VEGF/VEGFR2 Axis in Periodontal Disease Progression and Angiogenesis: Basic Approach for a New Therapeutic Strategy," In Vivo, vol. 30, no. 1, pp 53-60, 2016.

[13] P. Prakash, S. Rath, M. Mukherjee, A. Malik, D. Boruah, N.K. Sahoo, V. Dutta V, “Comparative evaluation of the marginal gingival epithelium in smokers and nonsmokers: a histomorphometric and immunohistochemical study," Int J Periodontics Restorative Dent, vol. 4, no. 6, pp. 781-786, 2014.

[14] G. Calsina, J.M. Ramón, J.J. Echeverría, “Effects of smoking on periodontal tissues,” J Clin Periodontol, vol. 29, no. 8, pp. 771-776, 2002.

[15] P.M. Preshaw, K. Henne, J.J. Taylor, R.A. Valentine, G. Conrads, “Age-related changes in immune function (immune senescence) in caries and periodontal diseases: a systematic review," J Clin Periodontol, vol. 44, no. 8, pp. S153-S177, 2017.

[16] J. Lindhe, N.P. Lang, T. Karring, T. Berglundh, W.V. Giannobile, "Clinical periodontology and implant dentistry," Fifth Edition, Blackwell, 2008.

[17] M. Sreedevi, A. Ramesh, C. Dwarakanath, "Periodontal status in smokers and nonsmokers: a clinical, microbiological, and histopathological study," Int J Dent, vol. 2012, Article ID 571590, pp. $1-10,2012$.

[18] H.J. Shiau, M.A. Reynolds, "Sex differences in destructive periodontal disease: exploring the biologic basis,” J Periodontol, vol. 81, no. 11, pp. 1505-1517, 2010. 